\title{
Portable LED-induced autofluorescence spectroscopy for oral cancer diagnosis
}

Yung-Jhe Yan

Ting-Wei Huang

Nai-Lun Cheng

Yao-Fang Hsieh

Ming-Hsui Tsai

Jin-Chern Chiou

Jeng-Ren Duann

Yung-Jiun Lin

Chin-Siang Yang

Mang Ou-Yang 


\title{
Portable LED-induced autofluorescence spectroscopy for oral cancer diagnosis
}

\author{
Yung-Jhe Yan, ${ }^{a}$ Ting-Wei Huang, ${ }^{b}$ Nai-Lun Cheng, ${ }^{c}$ Yao-Fang Hsieh, ${ }^{d}$ Ming-Hsui Tsai, ${ }^{e}$ Jin-Chern Chiou, ${ }^{a, b, e}$ \\ Jeng-Ren Duann, ${ }^{e}$ Yung-Jiun Lin, ${ }^{e}$ Chin-Siang Yang, ${ }^{b}$ and Mang Ou-Yang ${ }^{a, b, *}$ \\ ${ }^{a}$ National Chiao-Tung University, Institute of Electrical Control Engineering, Hsinchu City, Taiwan \\ ${ }^{b}$ National Chiao-Tung University, Department of Electrical and Computer Engineering, Hsinchu City, Taiwan \\ 'National Chiao-Tung University, Institute of Biomedical Engineering, Hsinchu City, Taiwan \\ dNational Central University, Department of Optics and Photonics, Jhongli City, Taoyuan County, Taiwan \\ ${ }^{\mathrm{e}}$ China Medical University, School of Medicine, Taichung City, Taiwan
}

\begin{abstract}
Oral cancer is a serious and growing problem in many developing and developed countries. To improve the cancer screening procedure, we developed a portable light-emitting-diode (LED)-induced autofluorescence (LIAF) imager that contains two wavelength LED excitation light sources and multiple filters to capture ex vivo oral tissue autofluorescence images. Compared with conventional means of oral cancer diagnosis, the LIAF imager is a handier, faster, and more highly reliable solution. The compact design with a tiny probe allows clinicians to easily observe autofluorescence images of hidden areas located in concave deep oral cavities. The ex vivo trials conducted in Taiwan present the design and prototype of the portable LIAF imager used for analyzing 31 patients with 221 measurement points. Using the normalized factor of normal tissues under the excitation source with $365 \mathrm{~nm}$ of the central wavelength and without the bandpass filter, the results revealed that the sensitivity was larger than $84 \%$, the specificity was not smaller than over $76 \%$, the accuracy was about $80 \%$, and the area under curve of the receiver operating characteristic (ROC) was achieved at about $87 \%$, respectively. The fact shows the LIAF spectroscopy has the possibilities of ex vivo diagnosis and noninvasive examinations for oral cancer. @ 2017 Society of Photo-Optical Instrumentation Engineers (SPIE) [DOI: 10.1117/1.JBO.22.4.045007]
\end{abstract}

Keywords: autofluorescence; oral cancer; multispectrum; LED; medical and biological imaging; optical diagnosis; tissue diagnostics statics for medicine.

Paper 160600RR received Aug. 30, 2016; accepted for publication Mar. 7, 2017; published online Apr. 19, 2017.

\section{Introduction}

Oral cancer is a serious and growing problem in the world. Over $90 \%$ of oral caners are oral squamous cell carcinoma (OSCC) in oral mucosa. ${ }^{1,2}$ The remaining oral cancers are verrucous carcinoma, spindle cell carcinoma, adenosquamous carcinoma, adenoid cell carcinoma, basaloid squamous carcinoma, and mucoepidermoid carcinoma. There are three types of carcinoma including squamous cell carcinoma, verrucous carcinoma, and spindle cell carcinoma in our study. OSCC begins from premalignant squamous cells or potentially malignant squamous cells in a stratified squamous epithelium. According to the world health organization grading system, OSCC is classified into well-differentiated, moderately differentiated and poorly differentiated types based on the degree of cell differentiation. ${ }^{1}$ In histopathology, well-differentiated squamous cell carcinoma (SCC), which resembles more likely normal squamous epithelium, has the feature of high keratinization that forms in epithelial pearls, less atypical mitotic activity, and less syncytium. Poorly differentiated SCC, which is mainly composed with immature cells, has the features of low keratinization, high atypical mitosis, more cells or nuclei with pleomorphism, and more syncytium. Moderately differentiated has the features of distinct nuclear pleomorphism, abnormal mitotic activity, and minimal keratinization. ${ }^{1,2}$ Verrucous cell carcinoma is a variant of well-differentiated SCC. It has exophytic slow-growth of keratinized stratified squamous epithelium and locally

*Address all correspondence to: Mang Ou-Yang, E-mail: oym @ cn.nctu.edu.tw subepithelial growth in destructive and expansive types. ${ }^{1}$ Spindle cell carcinoma is a rare variant of SCC. $^{3}$ It is composed of in situ or invasive squamous cell carcinoma and malignant spindle cells that constitute a mesenchymal appearance, but it is of epithelial origin. ${ }^{4}$ In clinical and histological, verrucous hyperplasia is considered to resemble verrucous carcinoma or squamous carcinoma. Compared to the invasive growth of verrucous carcinoma, verrucous hyperplasia is a superficial growth adjacent to normal epithelium. ${ }^{5}$ OSCC or premalignant OSCC almost presents the lesions which are abnormal damaged tissue or change in the tissue in oral cavity. The most common early lesions of premalignant OSCC are oral leukoplakia, oral erythroplakia, and speckled leukoplakia. The symptomatic lesions of site distribution in OSCC are classified under T category of the tumor-node-metastasis classification for malignant tumors. T1 lesions are the diameter of lesions that are $2 \mathrm{~cm}$ or $<2 \mathrm{~cm}$. $\mathrm{T} 2$ lesions are of diameter between 2 to $4 \mathrm{~cm}$. T3 lesions are of diameter longer than $4 \mathrm{~cm}$. T4 means that the tumor invades adjacent tissues. T4a means that the tumor invades through a cortical bone, into extrinsic or deep muscle of the tongue and T4b means that the tumor invades masticator space, pterygoid plates, or skull base. ${ }^{2}$

Presently, increasingly more people are suffering from oral cancer, and it has become a serious health problem in many developing and developed countries. In 2008, 263,000 new cases of lip and oral cavity cancer were reported in the world, and 127,000 persons died of this disease. ${ }^{6}$ In 2012, 300,373 
new cases of this cancer were reported worldwide, and 145,353 consequent deaths occurred. The age-standardized rates of incidence and mortality of oropharyngeal cancer in 2012 were 4.0 and 1.9 per 100,000 persons, respectively. ${ }^{7}$ From 2008 to 2012 , the reported incidence and mortality rates of oral cancer in the United States were 11 and 2.5 cases per 100,000 persons annually, respectively. In 2012, 291,108 persons had oral cavity and pharynx cancers in the United States. From 2007 to 2011, in the United States, the incidence rates for oral cavity cancer was the 7 th and 13th most common cancer in men and women, respectively. ${ }^{8}$ An estimated 45,780 new cases of oral cavity and pharynx cancers were diagnosed in the United States in 2015, and 8650 deaths were estimated to be due to this disease. ${ }^{9}$ In addition, India, Melanesia, and Taiwan have high rates of oral cancer attributable to betel quid or betel nut chewing. ${ }^{10}$ According to a report of the Ministry of Health and Welfare of Taiwan, the mortality rate of oral cancer in 2013 was 21.4 per 100,000 persons. In Taiwan, oral cancer is ranked as the fifth leading cause of cancer-related death in men. ${ }^{11}$

The possible explanations for the increasing incidence of oral cancer are as follows. First, people have insufficient awareness of the risk factors for oral cancer, and health care workers can detect only limited signs and symptoms of oral cancer from conventional examinations. The visual inspection and digital palpation of oral lesions in conventional examinations are insufficient for diagnosing oral cancer. ${ }^{12-15}$ Conventional clinical examinations that involve detecting suspicious lesions with toluidine blue staining, brush cytology, tissue chemiluminescence, and autofluorescence have variable effectiveness. ${ }^{15,16}$ Second, patient habits, such as tobacco and alcohol consumption and betel nut chewing as a cultural practice, expose the mucosal lining to carcinogens. ${ }^{15-17}$ Third, biopsies of oral lesions for diagnosing oral cancer must be performed by specialists in hospitals. However, in developing countries, people living in low-resource regions have inadequate resources, specialists, and hospitals for examination, which can delay cancer diagnosis and lead to high mortality.

Health care workers worldwide can prevent 37,000 oral cancer deaths through simple oral visual screening annually. ${ }^{18}$ In the United States, the five-year survival rate for oral cancer is $59 \%$, one of the lowest among all major cancers. This rate is persistent at $\sim 50 \%$ to $55 \%$ because most patients are diagnosed at an advanced stage. ${ }^{19,20}$ The survival rate for oral cancer is $\sim 50 \%$, despite improvements in diagnosis and treatment. ${ }^{21,22}$ Because patients diagnosed with early lesions can be treated, oral cancer must be detected and managed early to reduce mortality and morbidity. ${ }^{12,23-26}$ Therefore, the early detection of oral cancer may be a favorable and cost-effective method to improve the survival and quality of life of patients with oral cancer. ${ }^{27}$ However, the conventional oral examination with the visual inspection and digital palpation of oral lesions is not an objective and reliable method to distinguish lesions in different stages, and it results in the delayed treatment of patients or the oral cancer progressing to a late stage. ${ }^{12,13,15,24}$ Therefore, technologies with high sensitivity and specificity have been developed to facilitate the early diagnosis and treatment of patients. ${ }^{27-31}$

Optical imaging assists clinicians in detecting oral cancer. Optical imaging systems have been proposed to record the spatial distribution of fluorescence images with specific emission and excitation wavelengths in oral tissues. These systems can noninvasively detect a region of the oral cavity mucosa to determine the changes associated with oral cancer. ${ }^{16,18,27,32-35}$ A handheld device, the VELscope, was proposed for clinicians to noninvasively direct the fluorescence images of oral cavity tissues. ${ }^{16}$ Roblyer ${ }^{36}$ proposed a simple objective method to classify regions of interest (ROIs) and evaluate the sensitivity and specificity relative to the histopathology of oral neoplastic lesions through multispectral digital microscopy. The results indicated that quantitative autofluorescence imaging can provide a noninvasive and objective method for detecting oral neoplasia. Rahman proposed a headlight system for clinicians to screen and detect oral cancer. This portable system is mounted on the observer's head and used in clinics in low-resource countries. Digital images of oral tissues captured in white-light and fluorescence imaging modes can be used for the incorporation of automated image analysis algorithms to improve oral cancer detection. ${ }^{27}$ The autofluorescence images observed by the eye through the VELscope show the characteristic region of fluorescence visualization loss to indicate the development and progression of oral neoplasia. ${ }^{30}$ Other hand-held devices based on the autofluorescence image are Identafi 3000 (DentalEZ Inc.) and EVINCE ${ }^{\circledR}$ (MMOptics, São Carlos, Brazil). Identafi 3000 uses fluorescence, fiber optics, and confocal microscopy to screen and observe lesions. ${ }^{37}$ EVINCE $^{\circledR}$ (MMOptics, São Carlos, Brazil) emits light with LEDs system into tissues and observes tissue autofluorescence through an optical filter. ${ }^{38}$ The several studies having trials on detecting OSCC and oral potentially malignant disorders (OPMD) using VELscope, Identafi, and EVINCE seem to agree with certain consistent results in individual research and show the possibilities of OSCC and OPMD early detection potentially with autofluorescence images. ${ }^{16,33,35,37-47}$

Some studies observed the spectra of autofluorescence on different anatomical sites and found some characteristics between different sites. De Veld et al. ${ }^{48}$ observed autofluorescence characteristics of different anatomical sites with seven wavelengths excitation light source for healthy oral mucosa. They found that the normalized spectra were similar for locations that did not include dorsal side of the tongue (DST) and the vermilion border (VB). Furthermore, the DST and the VB having a peak at about $635 \mathrm{~nm}$ were observed. Mallia et al. ${ }^{49}$ used laser-induced autofluorescence technology for early cancer detection. This group similarly found that not only the DST and the VB but also the lateral side of the tongue had a peak at about $635 \mathrm{~nm}$ with a 404-nm excitation laser diode. Because of the same peak in malignant lesions, they excluded these sites from the study and had a good result of detecting premalignant and malignant tissues.

To provide clinicians with an objective and reliable method for the accurate diagnosis of oral cancer in clinics or developing countries, a portable detection device is required. In this paper, we propose a portable LED-induced autofluorescence (LIAF) imager. In a previous study, we developed an embedded relay lens microscopic hyperspectral imaging (ERL-MHSI) system. This system can be utilized for identifying pathological changes and is advantageous for developing a portable LIAF imager. This ex vivo trial conducted in Taiwan shows the design and prototype of a portable LIAF imager used for analyzing 31 patients. Finally, the results were analyzed using two normalized factors, where specimens were illuminated at two excitation wavelengths, and images were captured with or without two filters.

\section{Principle and Theory}

Several studies have reported that autofluorescence imaging may detect premalignant and malignant oral tissues. ${ }^{16,34,50,51}$ 
A portion of photons is absorbed by fluorophores present in oral mucosal molecules when the mucosa is illuminated at blue and UV-visible wavelengths. The fluorophores emit photons with lower energy, and subsequently, the photons can be viewed as fluorescence from the oral mucosal surface. The reduced fluorophores include nicotinamide adenine dinucleotide and flavin adenine dinucleotide in the epithelium, and collagen and elastin crosslinks in the stroma have been reportedly associated with the development and progression of oral neoplasia. ${ }^{16,52,53}$

Studies have proposed various technologies for clinicians to detect fluorescence images and diagnose oral neoplasia. ${ }^{54,55}$ Optical spectroscopy has been proposed to assist in the diagnosis of oral lesions. ${ }^{19,33,56,57}$ We previously developed the ERLMHSI system (Fig. 1). ${ }^{58}$ This system provides high spectral and spatial resolutions of the spectrum, and the morphological diagnosis of cancer can be performed in the transmittance and fluorescence modes under illumination with halogen and xenon lamps, respectively. The fluorescence images of cells or tissues can be identified as normal or abnormal. In the previous study, we demonstrated the efficiency of the ERL-MHSI system by diagnosing early stage oral cancer in 20 mice in vitro. The spatial distribution of tissue fluorescence at specific excitation wavelengths can be recorded, and the correlation between the fluorescence intensities of normal and abnormal cells is analyzed for the early detection of oral cancer. The correlation function $c(f, g)$ is shown as Eq. (1),

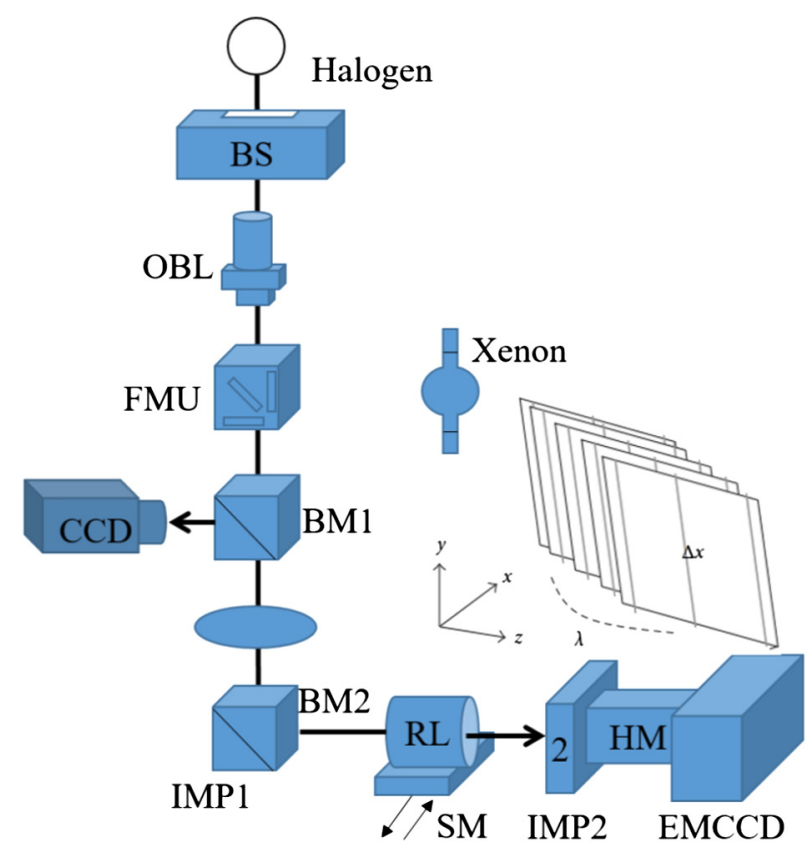

Fig. 1 The ERL-MHSI system has two light sources: halogen and xenon. The light passes through the biopsy stage, and the objective lens forms and magnifies the image of the ROI. In the transmittance mode, the fluorescent mirror unit does not open. Beam splitter 1 separates the light into two paths. The CCD immediately captures the biopsy image, and users can adjust the BS to determine the ROI of the biopsy. Beam splitter 2 guides the light toward the RL. The $R L$ projects the image from imaging plane 1 to imaging plane 2, which is the slit of the hyperspectrometer, and allows capturing an IMP2 image one line at a time on the electron multiplying chargecoupled device. Subsequently, the SM moves one step along the $x$-axis to capture the next line image of the slit and its spectrum. While the stepping motor scans along the $x$-axis, a separate line image is recorded on the $y-\lambda$ plane of the EMCCD. ${ }^{58}$ $c(f, g)=\int f(\lambda) \mathbb{d} \lambda \otimes \int g(\lambda) \mathbb{d} \lambda$

where $f(\lambda)$ and $g(\lambda)$ are the fluorescence intensities of normal and abnormal cells, and $\lambda$ is the wavelength. The fractal dimension is utilized to analyze the differences in morphological information between normal tissue and squamous cell carcinoma. Therefore, the ERL-MHSI system can serve as a platform for exploring specific excitation wavelengths and filters for oral cancer detection.

Fluorescence staining is widely used in biochemical invasive biopsy to enhance fluorescence images; however, autofluorescence is a more favorable method for in vivo examination. The ERL-MHSI system is large, and hyperspectral image data are extensive. Moreover, only in vitro trials can be conducted with the ERL-MHSI system. Therefore, the LIAF imager is proposed as a portable device that can be used in vivo. Based on the ERL-MHSI system, the design of a portable LIAF imager with specific excitation wavelengths and specific filters is introduced in the next section.

\section{Design and Embodiment}

The proposed portable LIAF imager contains two key components: two wavelengths LED excitation light sources to induce autofluorescence and multiple filters for capturing specific fluorescence images. Figure 2 shows a block diagram of the system, and Fig. 3 shows the construction of the LIAF imager. The LED light source in the imager is used for excitation. A rotary filter ring is integrated with the detector head for users to select the bandpass filter of interest. The probe in front of the LED light source is used for fixing the object distance and blocking ambient light. The filter ring behind the LED light source can be rotated by the fingers to change filters, and the filter location is fixed by positioning beads. The excitation light emitted from the

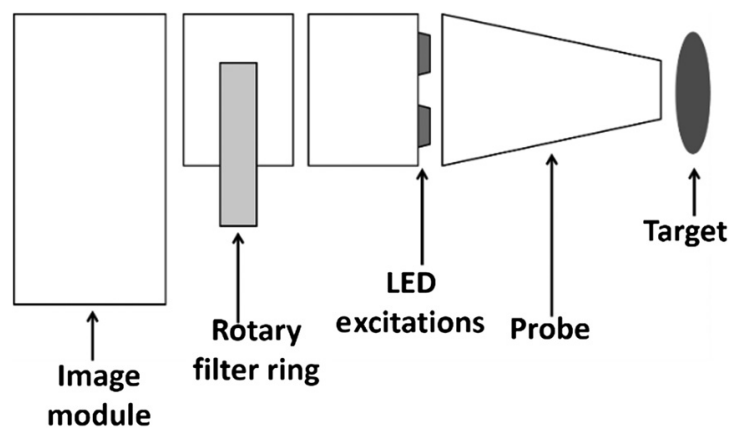

Fig. 2 Block diagram of the portable LIAF imager.

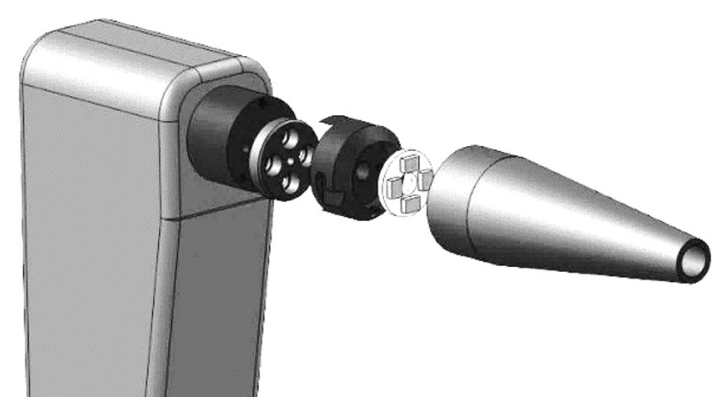

Fig. 3 Construction of the portable LIAF imager. 


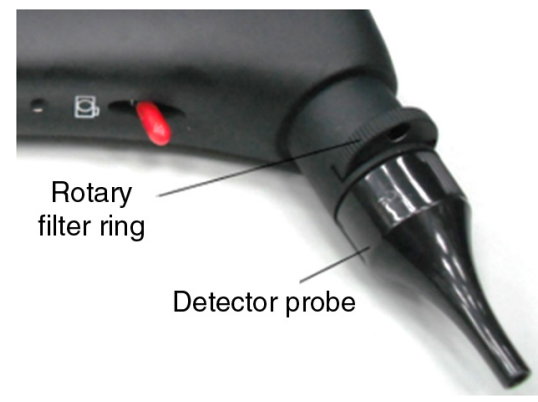

Fig. 4 Portable LIAF imager.

light source is projected on the target on the oral mucosal surface; autofluorescence is subsequently induced from this surface and passes through the probe. The light source has a hole in the middle (Fig. 3). Finally, the imager contains a lens, and a color complementary metal-oxide semiconductor (CMOS) captures the fluorescence images. Figure 4 shows an embodiment of the portable LIAF imager.

The LED light source has two excitation wavelength regions: 350 to $380 \mathrm{~nm}$ (L1) and 390 to $425 \mathrm{~nm}$ (L2). The rotary filter ring contains three bandpass filters, with wavelength regions of $\sim 490$ to $590 \mathrm{~nm}$ (F_I), 590 to $690 \mathrm{~nm}$ (F_II), and 650 to $750 \mathrm{~nm}$ (F_III). The resolution of the imaging module is $1600 \times 1200$. In Fig. 5(a), red (R), green (G), and blue (B) spectral responses of the CMOS are marked as a solid line, and the transmittance of the bandpass filters is marked as dashed lines. Figure 5(b) shows the combinations of the RGB responses and transmittance of the filters. The combinations of $\mathrm{G} \times \mathrm{FI}, \mathrm{R} \times \mathrm{FII}$, and $\mathrm{R} \times \mathrm{FIII}$ were larger than those of other combinations, and their bandwidths were less than $100 \mathrm{~nm}$. According to a screening result of the ERL-MHSI system, the representative autofluorescence spectra of normal and tumor tissues under L1 exciting light source have the peaks and gaps at about 525, 635, and $695 \mathrm{~nm}$. Therefore, these three bands were chosen to be observed.

\section{Materials and Methods}

The study was conducted at China Medical University Hospital, Taichung City, Taiwan. This study recruited patients who were referred to the Department of Otolaryngology-Head and Neck Surgery at the mentioned hospital because of suspicious oral lesions or those who were waiting to undergo head and neck surgery in the hospital ward. The study was reviewed and approved by the Institutional Review Board of China Medical

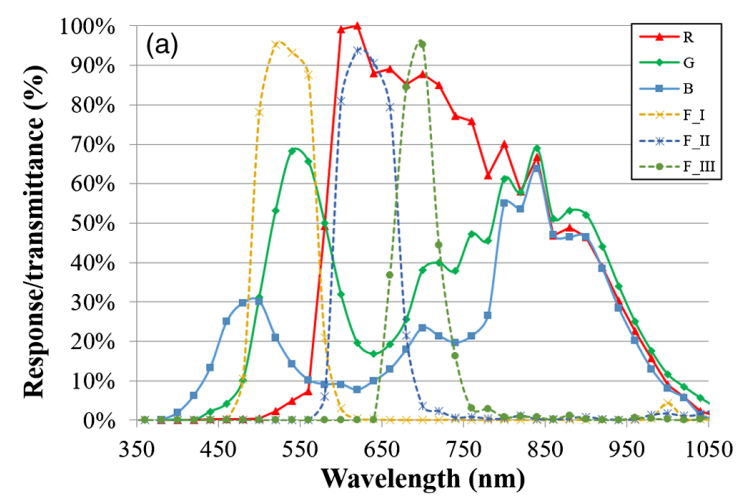

Table 1 The characteristic of 31 subjects.

\begin{tabular}{lcc} 
Characteristic & Male & Female \\
\hline Number & 30 & 1 \\
Age, mean \pm SD & $54.9 \pm 11.73$ & $76 \pm 0$ \\
\hline
\end{tabular}

University Hospital, and the imaging analysis was reviewed and approved by the Department of Electrical and Computer Engineering, National Chiao-Tung University, Taiwan. Written informed consent was obtained from all patients. Patients in the region who were aged 20 to 100 years and were scheduled for surgery to remove a previously diagnosed oral squamous carcinoma were eligible to participate in the study (Table 1).

The patients underwent standard-of-care surgery to remove oral neoplastic lesions. After surgical resection, the resected specimens were recorded using a camera. The specimens were obtained from the patients with squamous cell carcinomas after surgery and were transferred to the Biomedical Engineering R\&D Center, China Medical University Hospital.

Thirty-one specimens from the subject's oral cavity lesions of the 31 patients were surgically obtained by clinicians. After the clinician excised the tumor from the patient, the clinician selected four to nine points in the normal and tumor regions in a specimen [Fig. 6(a)] and started to acquire images immediately. Each selected point was photographed with two excitation light wavelengths (L1 and L2) through three channels (bandpass
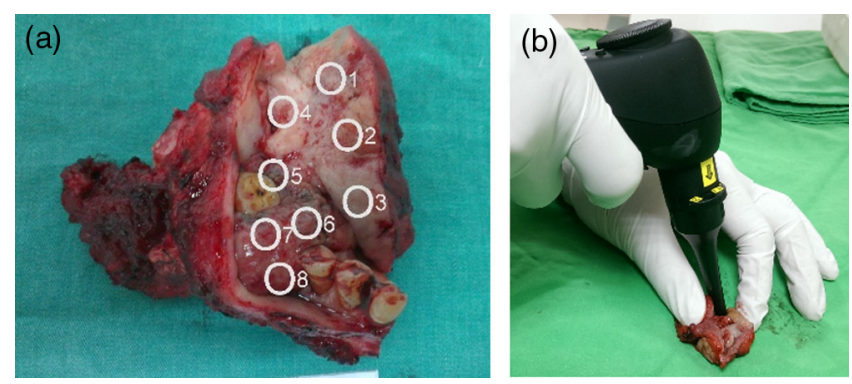

Fig. 6 Clinicians selected 4 to 9 points as measurement points depending on the area of the specimen, (a) the selection of eight measurement points on this specimen are marked with "o," and (b) each of the selected points will be captured at two excitation light source (L1 and L2) through three channels (bandpass filter F_1, F_2, and F_3) and a channel without filter.

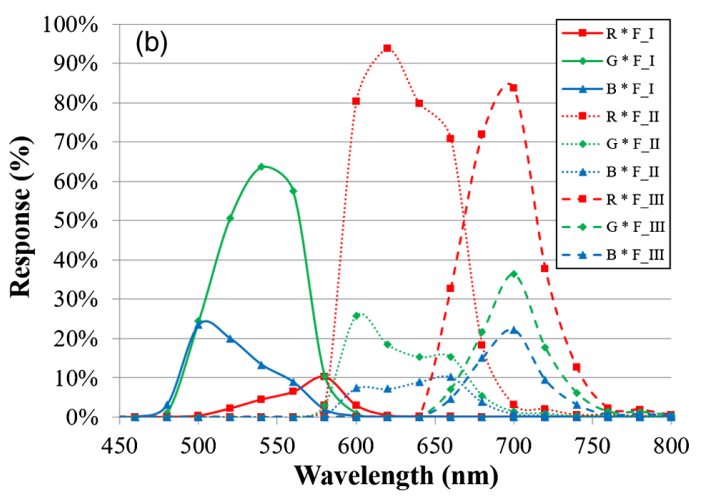

Fig. 5 (a) RGB responses of the CMOS and the transmittance of three bandpass filters and (b) the combinations of CMOS RGB responses and the transmittance of the three filters. 
filters: $F \_1, F \_2$, and $F \_3$ ) and a channel without the filters [Fig. 6(b)].

\section{Results and Analysis}

Tables 2 and 3 summarize the anatomical sites and histopathological diagnoses of the 31 specimens with 228 measurement points. The anatomical sites were located in the buccal cavity $(n=12)$, gum $(n=5)$, tongue $(n=8)$, lip $(n=2)$, and palate $(n=4)$. A total of 117 and 111 measurement points were imaged from the normal and tumor regions, respectively.

The experimental data analysis procedure is shown in Fig. 7. First, the effects of dark current noise were removed if the CMOS sensor detected the intensity of the dark current effects under a probe without any excitation. The images were captured from the normal and tumor regions of the specimen. Second, because of the limitation of the probe range, the ROIs of both normal and tumor images inside the probe range were selected [Fig. 6(a)]. Each intensity value of ROI in the captured images was calculated, and the values were normalized as red, green, and blue intensities as well as red-to-green, red-to-blue, or green-to-blue ratios of the ROI. Third, the mean value of ROI in normal or tumor images was the normalized factor. Fourth, each value of ROI was normalized by the normal and tumor factors, respectively. Based on the data in the last step, a scatter plot was plotted, and the Gaussian distribution of normal and tumor

Table 2 Anatomic sites of the 31 subjects.

\begin{tabular}{lccc}
\hline Anatomic site & $\begin{array}{c}\text { Specimen } \\
\text { number }\end{array}$ & $\begin{array}{c}\text { Measurement } \\
\text { point number of } \\
\text { normal region }\end{array}$ & $\begin{array}{c}\text { Measurement } \\
\text { point number of } \\
\text { tumor region }\end{array}$ \\
\hline Buccal & 12 & 45 & 44 \\
Gum & 5 & 19 & 19 \\
Tongue & 8 & 33 & 29 \\
Lips & 2 & 5 & 5 \\
Palate & 4 & 15 & 14 \\
Total & 31 & 117 & 111 \\
\hline
\end{tabular}

Table 3 Histopathological diagnoses of the 31 anatomic sites.

\begin{tabular}{|c|c|c|c|}
\hline Diagnosis & $\begin{array}{c}\text { Specimen } \\
\text { number }\end{array}$ & $\begin{array}{l}\text { Measurement } \\
\text { point number of } \\
\text { normal region }\end{array}$ & $\begin{array}{l}\text { Measurement } \\
\text { point number of } \\
\text { tumor region }\end{array}$ \\
\hline $\begin{array}{l}\text { Squamous cell } \\
\text { carcinoma }\end{array}$ & 26 & 97 & 92 \\
\hline $\begin{array}{l}\text { Spindle cell } \\
\text { carcinoma }\end{array}$ & 1 & 4 & 4 \\
\hline $\begin{array}{l}\text { Verrucous } \\
\text { carcinoma }\end{array}$ & 2 & 8 & 7 \\
\hline $\begin{array}{l}\text { Verrucous } \\
\text { hyperplasia }\end{array}$ & 2 & 8 & 8 \\
\hline Total & 31 & 117 & 111 \\
\hline
\end{tabular}

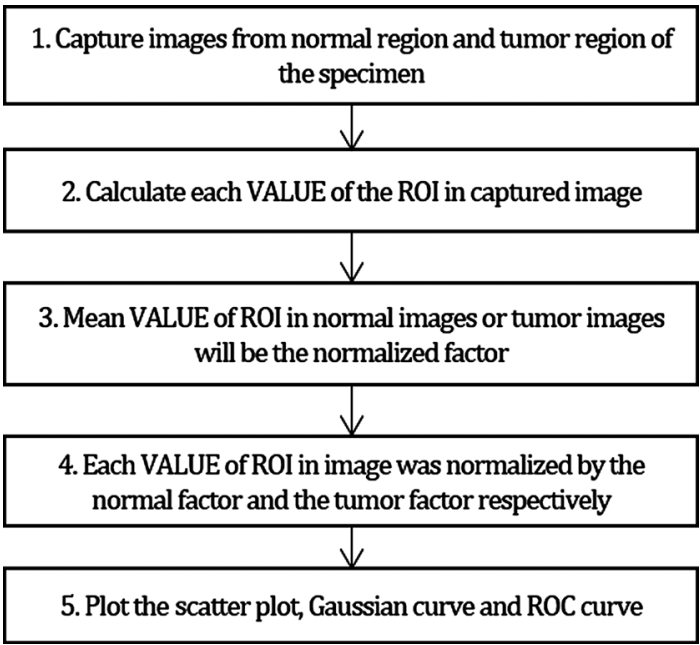

Fig. 7 The procedure to analyze the experimental data.

tissues was evaluated. Finally, the receiver operating characteristic (ROC) curve was plotted with various thresholds, where the $x$ - and $y$-axes of the ROC denote specificity (\%) and sensitivity (\%), respectively. The sensitivity, specificity, and accuracy were determined at various thresholds.

Figure 8 shows representative images of tumor and normal tissues illuminated with L1 and L2 with or without the filters. The images without the filters were clearer than those with the filters because the filters block fluorescence into the CMOS.

Some images were captured incorrectly because the investigator was removed out the analysis procedure. The valid images of normal and tumor tissues illuminated with both L1 and L2 were captured without the filters for 221 and 191 measurement points from 31 and 30 specimens, respectively. The valid images of normal and tumor tissues illuminated with L2 alone were captured with the filters F_I and F_II for 196 measurement points from 30 specimens and 189 measurement points from 30 specimens.

Data on the blue intensity of normal and tumor tissues illuminated with L1 without the filters are used to represent the result of the analysis. The scatter plot of this case (Fig. 9) was evaluated by using the first five steps of the analysis procedure.

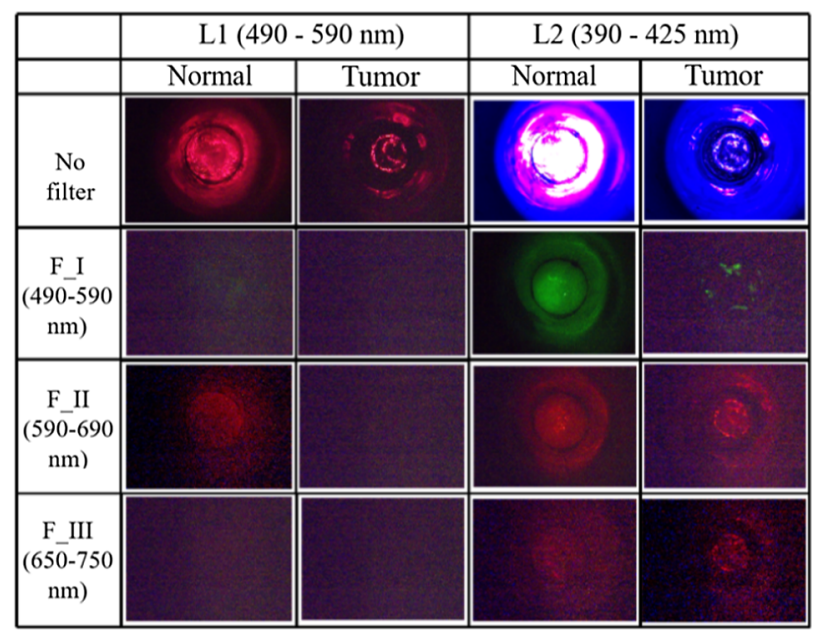

Fig. 8 The representative images of tumor tissues $(T)$ and normal tissues $(\mathrm{N})$ illuminated with $\mathrm{L} 1$ and $\mathrm{L} 2$ excitations without or with the bandpass filter F_1, F_2, or F_3. 


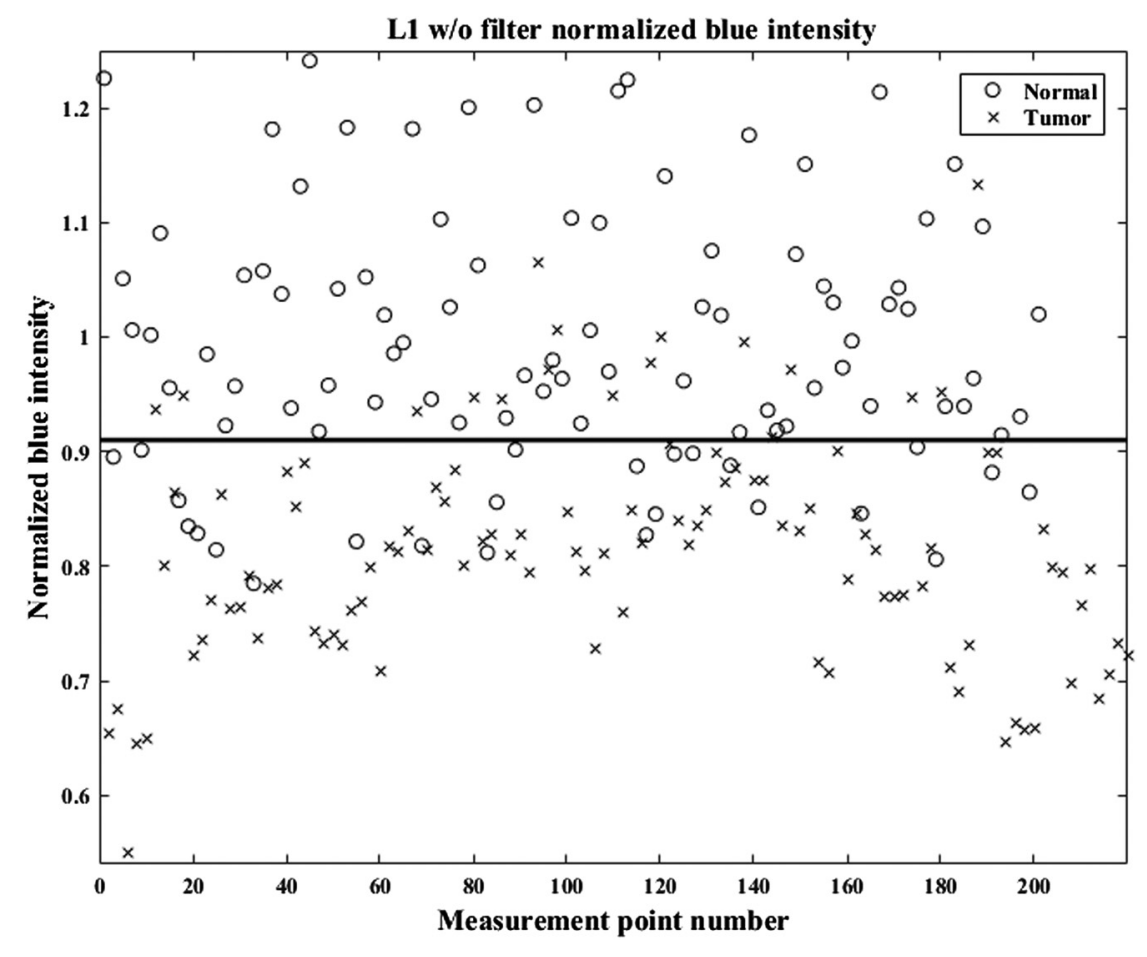

Fig. 9 Scatter plot of the blue intensity of tumor and normal tissues illuminated with L1 excitations without the filters. The 218 measurement points of ROI in normal region or tumor region are marked as "o" and " $x$," respectively. The solid line on scatter plot representing the threshold is defined by the crossing point between the Gaussian distribution of normal tissue and the Gaussian distribution of tumor tissue.

The 218 measurement points of the ROI in normal and tumor regions are marked as "o" and "x," respectively. The $P$ values of Kolmogorov-Smirnov (KS) tests for both normal and tumor regions exceeded 0.05 . Hence, the data distribution was similar to a Gaussian distribution. Moreover, the KS tests were performed for tissues illuminated with other excitation wavelengths with other filters, and all data distributions were similar to a Gaussian distribution. In Fig. 9, the solid line on the scatter

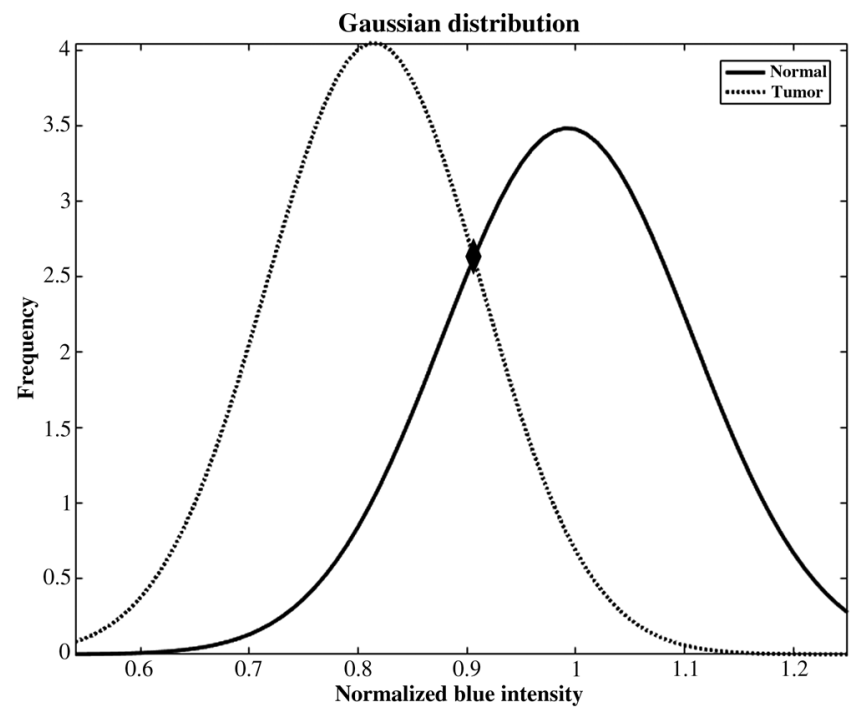

Fig. 10 Gaussian distribution of the blue intensity of normal and tumor tissues illuminated with L1 excitations without the filters. The threshold is defined by the crossing point marked as "filled diamond" between the Gaussian distribution of normal and tumor tissues drawn as solid line and dotted line, respectively. plot represents the threshold defined by the crossing point marked as "filled diamond" between the Gaussian distribution of normal and tumor tissues drawn as a solid line and dotted line, respectively (Fig. 10). The threshold value in this case was 0.91 . Most data on tumor tissues are located under the solid threshold line (Fig. 9).

The best case showed that sensitivity was $84.68 \%$, specificity was $76.24 \%$, and accuracy was $80.66 \%$. The solid line in Fig. 11 shows the ROC, and the area under the curve (AUC) of the ROC was $87.56 \%$. In a similar analysis procedure, the valid images were analyzed, and the results are presented in Table 4, where NF_T and NF_N indicate the normalized factors for tumor and normal tissues, respectively. The AUC is a quantitative index for estimating the performance of the specific excitation wavelengths and filters. Table 5 shows the discrimination by the

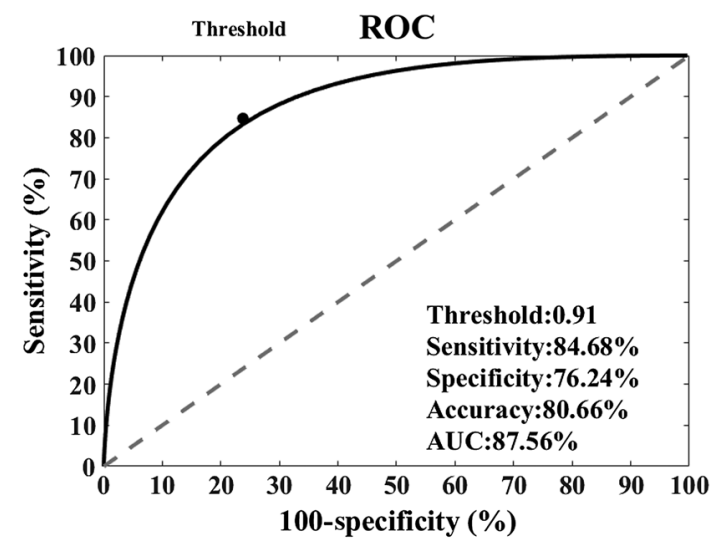

Fig. 11 ROC curve of blue intensity illuminated with L1 excitations without the filters. 
Table 4 The results of six types analysis of L1 and L2 excitation without the filter and L2 excitation with F_I filter and F_II filter, where NF_T means the normalized factor of tumor tissues and NF_N means the normalized factor of normal tissues.

\begin{tabular}{|c|c|c|c|c|c|c|c|c|c|}
\hline & & \multicolumn{2}{|c|}{ L1 } & \multicolumn{2}{|c|}{ L2 } & \multicolumn{2}{|c|}{ L2_F_I } & \multicolumn{2}{|c|}{ L2_F_II } \\
\hline & & $\mathrm{NF}_{-} \mathrm{T}$ & $N F \_N$ & NF_t & $\mathrm{NF} \_\mathrm{N}$ & NF_T & $N F \_N$ & $N F_{-} T$ & $\mathrm{NF} \_\mathrm{N}$ \\
\hline \multirow[t]{5}{*}{$R$} & Threshold & 1.29 & 0.80 & 1.47 & 0.84 & 0.97 & 0.97 & 1.09 & 0.89 \\
\hline & $\mathrm{Se}(\%)$ & 96.40 & 81.98 & 96.00 & 85.00 & 91.35 & 15.38 & 83.50 & 25.24 \\
\hline & $\mathrm{Sp}(\%)$ & 60.00 & 72.28 & 56.04 & 63.74 & 21.21 & 89.13 & 26.74 & 88.37 \\
\hline & Accuracy (\%) & 78.28 & 77.36 & 76.96 & 74.87 & 57.14 & 50.00 & 57.67 & 53.97 \\
\hline & AUC (\%) & 85.19 & 83.91 & 87.57 & 80.03 & 50.73 & 52.75 & 60.51 & 63.63 \\
\hline \multirow[t]{5}{*}{$\mathrm{G}$} & Threshold & 1.13 & 0.87 & 1.73 & 0.87 & 1.24 & 0.82 & 1.08 & 1.08 \\
\hline & $\mathrm{Se}(\%)$ & 90.99 & 45.95 & 97.00 & 85.00 & 95.19 & 69.23 & 93.20 & 19.42 \\
\hline & $\mathrm{Sp}(\%)$ & 41.67 & 82.86 & 48.35 & 53.85 & 51.09 & 71.74 & 16.28 & 94.19 \\
\hline & Accuracy (\%) & 66.67 & 63.89 & 73.82 & 70.16 & 74.49 & 70.41 & 58.20 & 53.44 \\
\hline & AUC (\%) & 67.63 & 67.57 & 89.94 & 73.38 & 85.05 & 77.54 & 57.08 & 51.37 \\
\hline \multirow[t]{5}{*}{ B } & Threshold & 1.09 & 0.91 & 1.20 & 0.85 & 0.97 & 1.04 & 0.97 & 1.04 \\
\hline & $\mathrm{Se}(\%)$ & 92.79 & 84.68 & 94.00 & 65.00 & 91.35 & 21.15 & 88.35 & 38.83 \\
\hline & $\mathrm{Sp}(\%)$ & 69.52 & 76.24 & 68.13 & 90.11 & 23.91 & 88.04 & 38.37 & 91.86 \\
\hline & Accuracy (\%) & 81.48 & 80.66 & 81.68 & 76.96 & 59.69 & 52.55 & 65.61 & 62.96 \\
\hline & AUC & 87.32 & 87.56 & 84.70 & 83.29 & 52.69 & 55.08 & 65.50 & 68.30 \\
\hline \multirow[t]{5}{*}{$\mathrm{R} / \mathrm{G}$} & Threshold & 1.21 & 0.83 & 1.31 & 1.38 & 0.85 & 1.44 & 1.12 & 0.85 \\
\hline & $\mathrm{Se}(\%)$ & 90.09 & 76.58 & 93.00 & 26.00 & 89.42 & 36.54 & 86.41 & 25.24 \\
\hline & Sp (\%) & 58.33 & 72.82 & 23.08 & 92.31 & 58.70 & 93.48 & 27.91 & 90.70 \\
\hline & Accuracy (\%) & 74.43 & 74.77 & 59.69 & 57.59 & 75.00 & 63.27 & 59.79 & 55.03 \\
\hline & AUC (\%) & 85.00 & 82.42 & 58.47 & 61.60 & 80.97 & 80.42 & 58.03 & 59.52 \\
\hline \multirow[t]{5}{*}{$\mathrm{R} / \mathrm{B}$} & Threshold & 1.18 & 0.84 & 1.34 & 0.85 & 1.04 & 0.96 & 1.11 & 0.86 \\
\hline & $\mathrm{Se}(\%)$ & 92.79 & 69.37 & 96.00 & 58.00 & 91.35 & 27.88 & 82.52 & 32.04 \\
\hline & $\mathrm{Sp}(\%)$ & 56.31 & 85.58 & 42.86 & 64.84 & 21.74 & 90.22 & 26.74 & 89.53 \\
\hline & Accuracy (\%) & 75.23 & 77.21 & 70.68 & 61.26 & 58.67 & 57.14 & 57.14 & 58.20 \\
\hline & AUC & 80.55 & 78.57 & 81.75 & 64.30 & 53.84 & 55.47 & 63.85 & 64.96 \\
\hline \multirow[t]{5}{*}{$\mathrm{G} / \mathrm{B}$} & Threshold & 0.94 & 1.11 & 1.61 & 0.70 & 1.25 & 0.82 & 1.08 & 0.97 \\
\hline & Se (\%) & 73.87 & 42.34 & 98.00 & 52.00 & 95.19 & 66.35 & 94.17 & 49.51 \\
\hline & $\mathrm{Sp}(\%)$ & 57.66 & 88.07 & 35.16 & 67.03 & 51.09 & 70.65 & 26.74 & 67.44 \\
\hline & Accuracy (\%) & 65.77 & 65.00 & 68.06 & 59.16 & 74.49 & 68.37 & 63.49 & 57.67 \\
\hline & AUC (\%) & 68.94 & 64.30 & 86.59 & 57.31 & 84.73 & 76.75 & 61.05 & 57.35 \\
\hline
\end{tabular}

AUC that occurs in different ranges. ${ }^{57}$ Some sensitivities based on the tumor normalization factor exceeded $80 \%$. The AUC in 18 cases was higher than $80 \%$, presented in bold in Table 4 . The illumination of tissues with L1 without the filters resulted in the better diagnostic accuracy for oral cancer. Furthermore, we randomly picked five points to do the blind test for the best case. The threshold was determined by other unpicked measurement points in the same case. Table 6 shows the result of the blind test. The sensitivity, specificity, and accuracy were the same in $100 \%$. The AUC of the ROC was about $81 \%$. 
Table 5 AUC performance definition.

\begin{tabular}{lc}
$\mathrm{AUC}=0.5$ & No discrimination \\
$0.7 \leqq \mathrm{AUC}<0.8$ & Acceptable \\
$0.8 \leqq \mathrm{AUC}<0.9$ & Excellent discrimination \\
$\mathrm{AUC} \geqq 0.9$ & Outstanding discrimination \\
\hline
\end{tabular}

Table 6 The result of blind test with L1 excitation without the filter in blue intensity.

Threshold

0.92

Se $(\%)$

100

Sp $(\%)$

100

Accuracy (\%)

100

AUC (\%)

81.6889919

\section{Discussion}

This paper proposes a portable LIAF imager composed of a two wavelength LED excitation light source and multiple filters for oral cancer detection. Combinations of LED wavelengths and filter transmittance in the LIAF imager were explored using the ERL-MHSI system. Because this system is large and involves extensive hyperspectral imaging data, the LIAF imager is proposed as a compact device for in vitro application. It has a probe for fixing the objective length, thus providing protection from ambient light. Clinicians can easily observe the autofluorescence images of the hidden area located in a concave deep oral cavity, such as the deep port between the gums, gingiva, and mouth lining, by using the probe. In addition, VELscope, Identafi 3000, EVINCE, and the LIAF imager devices using different wavelength light sources and bandpass filters are according to the results of their studies. The efficiency of using different wavelength light sources or combined with bandpass filters for early detection needs more examination and studies for evidence. VELscope, Identafi 3000, and EVINCE mainly depend on fluorescence visualization of human eyes. Differently, LIAF is using RGB CMOS in order to acquire the quantitative data of the autofluorescence images. Therefore, the data are stored in a database and used to develop and improve the algorithm regarding early oral cancer analysis and detection.

Because of their betel nut chewing habit, patients in Asia have a higher risk of oral cancer than do those in the United States. Therefore, it is necessary to conduct similar ex vivo trials of oral cancer detection in Asia. In this study, 31 specimens from Taiwan were analyzed, and all sites were defined through biopsy and pathology. We analyzed two normalized factors under two excitation wavelengths with and without filters. The case with a normalized factor for normal tissue under L1 without a filter showed that sensitivity was larger than $84 \%$, the specificity was not smaller than over $76 \%$, the accuracy was about $80 \%$, and the AUC of ROC curve was achieved at about $87 \%$, respectively. Sensitivities exceeded $80 \%$ under other conditions when a tumor normalization factor was used (Table 4); the AUC in 18 cases exceeded $80 \%$ (marked in bold). The illumination of tissues with L1 without the filters resulted in better accuracy for oral cancer. If the AUC of the cases is lower than $70 \%$, this case is not considered to be effective or usable. The AUC of almost low specificity cases were lower than $70 \%$. However, the reason for low specificity for some cases needs to be further discussed.

In future studies, the excitation intensity of the LED light source must not only enhance the detection of oral lesions, but also modify the wavelength region of the excitation light and filters. Ex vivo trials will be implemented steadily to increase the number of subjects for increasing sensitivity, specificity, accuracy, and AUC. Moreover, on the basis of previous studies that used the ERL-MHSI system because of its higher magnification ability, oral cancer can be morphologically identified in the future.

\section{Disclosures}

The authors declare no conflicts of interest, financial or otherwise.

\section{Acknowledgments}

This work was partially financial supported by the Ministry of Science and Technology of Taiwan (MOST) (MOST 104-2622E-009-015-CC2, MOST 105-2623-E-009-008-D, MOST 1052221-E-009-082), the National Chiao-Tung University and China Medical University. The authors would like to thank Dr. Ming-Hsui Tsai and Prof. Chia-Ing Jan from China Medical University Hospital for experimental assistance. Prof. MingHsui Tsai helped and carried out the ex vivo trials. Prof. Chia-Ing Jan supported and provided the pathological analysis.

\section{References}

1. T. Kirita and K. Omura, Eds., Oral Cancer Diagnosis and Therapy, pp. 1-52, Springer, Japan (2015).

2. K. U. E. Ogbureke, Eds., Oral Cancer, p. 3, InTech, Rijeka (2016).

3. M. V. Biradar et al., "Spindle cell carcinoma of the tongue: a rare variant of squamous cell carcinoma," Ecancermedicalscience 8, 447 (2014).

4. N. Parikh and N. Desai, "Spindle cell carcinoma of the oral cavity: a case report of a rare entity and review of literature," J. Acad. Adv. Dent. Res. 2(2), 31-36 (2011).

5. V. K. Hazarey, S. M. Ganvir, and A. S. Bodhade, "Verrucous hyperplasia: a clinico-pathological study," J. Oral Maxillofac. Pathol. 15(2), 187-191 (2011).

6. J. Ferlay et al., "Estimates of worldwide burden of cancer in 2008: GLOBOCAN 2008," Int. J. Cancer 127, 2893-2917 (2010).

7. J. Ferlay et al., "Globocan 2012: estimated cancer incidence, mortality and prevalence worldwide in 2012," http://globocan.iarc.fr/Pages/fact_ sheets_population.aspx (29 August 2016).

8. N. Howlader et al., "SEER cancer statistics review, 1975-2012," http:// seer.cancer.gov/csr/1975_2012/ (29 August 2016).

9. American Cancer Society, "Cancer facts and figures 2015," http:// www.cancer.org/research/cancerfactsstatistics/cancerfactsfigures2015/ (29 August 2016).

10. D. M. Parkin et al., "Global cancer statistics, 2002," CA: Cancer J. Clin. 55, 74-108 (2005).

11. Ministry of Health and Welfare of Taiwan, "Oral cancer and leading cause of death," http://www.mohw.gov.tw/cht/DOS/DisplayStatistic File.aspx?d=45337 (29 August 2016).

12. C. S. Farah and M. J. McCullough, "A pilot case control study on the efficacy of acetic acid wash and chemiluminescent illumination (ViziLite $^{\mathrm{TM}}$ ) in the visualisation of oral mucosal white lesions," Oral Oncol. 43, 820-824 (2007).

13. M. A. Huber, S. A. Bsoul, and G. T. Terezhalmy, "Acetic acid wash and chemiluminescent illumination as an adjunct to conventional oral soft tissue examination for the detection of dysplasia: a pilot study," Quintessence Int. 35(5), 378-384 (2004). 
14. A. R. Kerr, D. A. Sirois, and J. B. Epstein, "Clinical evaluation of chemiluminescent lighting: an adjunct for oral mucosal examinations," J. Clin. Dent. 17(3), 59-63 (2006).

15. M. W. Lingen et al., "Critical evaluation of diagnostic aids for the detection of oral cancer," Oral Oncol. 44, 10-22 (2008).

16. P. M. Lane et al., "Simple device for the direct visualization of oralcavity tissue fluorescence," J. Biomed. Opt. 11(2), 024006 (2006).

17. D. P. Slaughter, H. W. Southwick, and W. Smejkal, “"Field cancerization' in oral stratified squamous epithelium. Clinical implications of multicentric origin," Cancer 6, 963-968 (1953).

18. R. Sankaranarayanan et al., "Effect of screening on oral cancer mortality in Kerala, India: a cluster-randomised controlled trial," The Lancet $\mathbf{3 6 5}$, 1927-1933 (2005).

19. R. A. Schwarz et al., "Autofluorescence and diffuse reflectance spectroscopy of oral epithelial tissue using a depth-sensitive fiber-optic probe," Appl. Opt. 47, 825-834 (2008).

20. B. W. Neville and T. A. Day, "Oral cancer and precancerous lesions," CA: Cancer J. Clin. 52, 195-215 (2002).

21. H. P. Howaldt, M. Frenz, and H. Pitz, "Results from DÖSAK observational studies," in Carcinoma of the Oral Cavity and Oropharynx, H. D. Pape, U. Ganzer, and G. Schmitt, Eds., pp. 173-182, Springer, Berlin Heidelberg (1994).

22. J. P. Shah and W. Lydiatt, "Treatment of cancer of the head and neck," CA: Cancer J. Clin. 45, 352-368 (1995).

23. F. Brunin et al., "Cancer of the base of the tongue: past and future," Head Neck 21, 751-759 (1999).

24. D. Maraki, J. Becker, and A. Boecking, "Cytologic and DNA-cytometric very early diagnosis of oral cancer," J. Oral Pathol. Med. 33, 398-404 (2004).

25. C. S. Farah et al., "Efficacy of tissue autofluorescence imaging (velscope) in the visualization of oral mucosal lesions," Head Neck 34, 856-862 (2012).

26. M. S. Rahman et al., "Evaluation of a low-cost, portable imaging system for early detection of oral cancer," Head Neck Oncol. 2, 10 (2010).

27. M. Rahman et al., "Low-cost, multimodal, portable screening system for early detection of oral cancer," J. Biomed. Opt. 13(3), 030502 (2008).

28. C. S. Farah and M. J. McCullough, "Oral cancer awareness for the general practitioner: new approaches to patient care," Australian Dent. J. 53, 2-10 (2008).

29. M. J. McCullough, G. Prasad, and C. S. Farah, "Oral mucosal malignancy and potentially malignant lesions: an update on the epidemiology, risk factors, diagnosis and management," Australian Dent. J. 55, 61-65 (2010).

30. L. L. Patton, J. B. Epstein, and A. R. Kerr, "Adjunctive techniques for oral cancer examination and lesion diagnosis: a systematic review of the literature," J. Am. Dent. Assoc. 139, 896-905 (2008).

31. M. P. Rethman et al., "Evidence-based clinical recommendations regarding screening for oral squamous cell carcinomas," J. Am. Dent. Assoc. 141, 509-520 (2010).

32. K. Onizawa et al., "Usefulness of fluorescence photography for diagnosis of oral cancer," Int. J. Oral Maxillofac. Surg. 28, 206-210 (1999).

33. C. S. Betz et al., "Autofluorescence imaging and spectroscopy of normal and malignant mucosa in patients with head and neck cancer," Lasers Surg. Med. 25, 323-334 (1999).

34. E. Svistun et al., "Vision enhancement system for detection of oral cavity neoplasia based on autofluorescence," Head Neck 26, 205-215 (2004).

35. C. F. Poh et al., "Direct fluorescence visualization of clinically occult high-risk oral premalignant disease using a simple hand-held device," Head Neck 29, 71-76 (2007).

36. D. Roblyer et al., "Multispectral optical imaging device for in vivo detection of oral neoplasia," J. Biomed. Opt. 13(2), 024019 (2008).

37. D. V. Messadi, "Diagnostic aids for detection of oral precancerous conditions," Int. J. Oral Sci. 5(2), 59-65 (2013).

38. L. E. Simonato et al., "Fluorescence visualization efficacy for detecting oral lesions more prone to be dysplastic and potentially malignant disorders: a pilot study," Photodiagn. Photodyn. Ther. 17, 1-4 (2017).

39. R. Nagi et al., "Efficacy of light based detection systems for early detection of oral cancer and oral potentially malignant disorders: systematic review," Med. Oral Patol. Oral Y Cirugia Bucal, 21(4), e447 (2016).

40. K. H. Awan, P. R. Morgan, and S. Warnakulasuriya, "Assessing the accuracy of autofluorescence, chemiluminescence and toluidine blue as diagnostic tools for oral potentially malignant disorders-a clinicopathological evaluation," Clin. Oral Invest. 19(9), 2267-2272 (2015).

41. M. Petruzzi et al., "Evaluation of autofluorescence and toluidine blue in the differentiation of oral dysplastic and neoplastic lesions from non dysplastic and neoplastic lesions: a cross-sectional study," J. Biomed. Opt. 19(7), 076003 (2014).

42. Y. Lalla, M. A. T. Matias, and C. S. Farah, "Assessment of oral mucosal lesions with autofluorescence imaging and reflectance spectroscopy," J. Am. Dent. Assoc. 147(8), 650-660 (2016).

43. M. Scheer et al., "Autofluorescence imaging in recurrent oral squamous cell carcinoma," Oral Maxillofac. Surg. 20(1), 27-33 (2016).

44. A. Ramanathan et al., "Utility of autofluorescence imaging in the detection of oral mucosal lesions in elderly institutionalised subjects," Ann. Dent. 21(1) (2015).

45. E. Jané-Salas et al. "Autofluorescence and diagnostic accuracy of lesions of oral mucosa: a pilot study," Brazilian Dent. J. 26(6), 580-586 (2015).

46. D. V. Messadi et al., "The clinical effectiveness of reflectance optical spectroscopy for the in vivo diagnosis of oral lesions," Int. J. Oral Sci. 6(3), 162-167 (2014).

47. B. H. Malik et al., "A novel multimodal optical imaging system for early detection of oral cancer," Oral Surg. Oral Med. Oral Pathol. Oral Radiol. 121(3), 290-300 (2016).

48. D. C. de Veld et al., "Autofluorescence characteristics of healthy oral mucosa at different anatomical sites," Lasers Surg. Med. 32(5), 367-376 (2003)

49. R. J. Mallia et al., "Laser-induced autofluorescence spectral ratio reference standard for early discrimination of oral cancer," Cancer 112(7), 1503-1512 (2008).

50. D. C. G. De Veld et al., "The status of in vivo autofluorescence spectroscopy and imaging for oral oncology," Oral Oncol. 41, 117-131 (2005).

51. U. Utzinger et al., "Optimal visual perception and detection of oral cavity neoplasia," IEEE Trans. Biomed. Eng. 50, 396-399 (2003).

52. A. Gillenwater et al., "Noninvasive diagnosis of oral neoplasia based on fluorescence spectroscopy and native tissue autofluorescence," Arch. Otolaryngol. Head Neck Surg. 124, 1251-1258 (1998).

53. M. G. Müller et al., "Spectroscopic detection and evaluation of morphologic and biochemical changes in early human oral carcinoma," Cancer 97, 1681-1692 (2003).

54. A. Gillenwater, V. Papadimitrakopoulou, and R. Richards-Kortum, "Oral premalignancy: new methods of detection and treatment," Curr. Oncol. Rep. 8, 146-154 (2006).

55. V. R. Kolli et al., "Native cellular fluorescence of neoplastic upper aerodigestive mucosa," Arch. Otolaryngol. Head Neck Surg. 121, 1287-1292 (1995).

56. J. K. Dhingra et al., "Early diagnosis of upper aerodigestive tract cancer by autofluorescence," Arch. Otolaryngol. Head Neck Surg. 122, 1181-1186 (1996).

57. S. K. Majumder et al., "Multi-class classification algorithm for optical diagnosis of oral cancer," J. Photochem. Photobiol., B 85, 109-117 (2006).

58. Y.-F. Hsieh et al., "Development of a novel embedded relay lens microscopic hyperspectral imaging system for cancer diagnosis: use of the mice with oral cancer to be the example," Int. J. Spectrosc. 2012, 710803 (2012).

Yung-Jhe Yan is a PhD student at the University of Electrical and Control Engineering. He shifted to Graduate Institute of Electrical Control Engineering in 2013, and he has been a master's student for two years. His research focuses on photonics system integrating and biomedical image processing.

Ting-Wei Huang received his PhD from the Department of Optics and Photonics at the National Central University in 2011. He is a postdoctoral researcher at National Chiao Tung University (NCTU) in Taiwan from 2011 to 2016. Currently, he is an optical engineer in a Taiwan company. His majors include color science, optical lens design, and biomedical application of optics and spectrum.

Ming-Hsui Tsai is currently a chairman and professor of the Department of Otolaryngology at China Medical University and Hospital. His expertise is in otolaryngology head and neck surgery. In his department, they discovered and managed over 500 new 
cases of head and neck cancer, and two-thirds of them are oral cancer. He has received grants from the National Science Council and National Health Research Institutes, and published more than 150 articles in peer-reviewed scientific or clinical journals and conference papers in the fields of otolaryngology and head and neck oncology.

Jin-Chern Chiou received his MS and PhD degrees in aerospace engineering science from the University of Colorado, Boulder, Colorado, USA, in 1986 and 1990, respectively. His research interests include micro-electro-mechanical systems, multibody dynamic systems, and biomedical engineering. He is currently a professor in the Department of Electrical and Computer Engineering, National Chiao Tung University, Hsinchu, Taiwan, and the deputy general director of Biomedical Technology and Device Research Laboratories, Industrial Technology Research Institute, Hsinchu, Taiwan.

Yung-Jiun Lin received his $\mathrm{PhD}$ in electrical and control engineering from National Chiao Tung University, Taiwan, in 2005. In 2006, he was working for the Micro-system and Control Laboratory and Brain Research Center, NCTU, as a postdoctoral researcher. In 2009, he was working for the Biomedical Engineering Research and Development Center at China Medical University Hospital, as a senior engineer. His research interests are design, application, and clinical trial of medical devices for unmet clinical needs.

Mang Ou-Yang received his MS and PhD degrees in electro-optical engineering from the National Chiao-Tung University, Taiwan, in 1993 and 1998, respectively. Currently, he is a professor of the Department of Electrical and Computer Engineering, National Chiao-Tung University. His research interests are related to photonic and industrial instrumentation development, including biomedical engineering, hyperspectroscopy, optical metrology and control, and color display engineering.

Biographies for the other authors are not available. 